\title{
EMG-Based Decoding of Grasp Gestures in Reaching-to-Grasping Motions
}

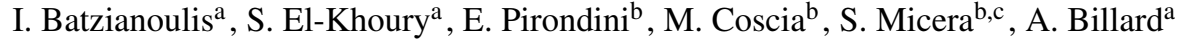 \\ ${ }^{a}$ Learning Algorithms and Systems Laboratory (LASA), Ecole Polytechnique Federale de Lausanne (EPFL), Switzerland \\ ${ }^{b}$ Translational Neural Engineering Lab, Ecole Polytechnique Federale de Lausanne (EPFL), Switzerland \\ ${ }^{c}$ Advanced Robotics Technology and Systems (ARTS) Laboratory, Scuola Superiore Sant' Anna, Pisa, Italy
}

\begin{abstract}
Predicting the grasping function during reach-to-grasp motions is essential for controlling a prosthetic hand or a robotic assistive device. An early accurate prediction increases the usability and the comfort of a prosthetic device. This work proposes an electromyographic-based learning approach that decodes the grasping intention at an early stage of reach-to-grasp motion, i.e. before the final grasp/hand pre-shape takes place. Superficial electrodes and a Cyberglove were used to record the arm muscle activity and the finger joints during reach-to-grasp motions. Our results showed a $90 \%$ accuracy for the detection of the final grasp about $0.5 \mathrm{sec}$ after motion onset. This paper also examines the effect of different objects' distances and different motion speeds on the detection time and accuracy of the classifier. The use of our learning approach to control a 16-degrees of freedom robotic hand confirmed the usability of our approach for the real-time control of robotic devices.
\end{abstract}

Keywords:

Reach-to-grasp, grasp planning, machine learning, electromyographic (EMG) signals, prosthesis

\section{Introduction}

Nowadays, robotic devices are frequently used to restore motor abilities lost after pathologies or trauma, such as exoskeletons and operative devices adopted for the patients assistance during rehabilitation, and the prostheses for amputees [1]. Studies on amputees and stroke patients [2, 3] have reported that comfort is one of the priorities for the acceptability of a wearable robotic device, and that inconvenient and time ineffective systems may avert individuals from using a prosthetic device [2]. To increase the level of comfort and effectiveness, these devices should detect the human intention early enough in order to ensure the smooth and prompt behavior of the system.

It has been extensively demonstrated that users motion intention can be accurately detected by surface electromyographic recordings (sEMG) [4]. Different

\footnotetext{
Email addresses: iason batzianoulisdepfl.ch (I. Batzianoulis $\left.^{a}\right)$, sahar.elkhourydepfl.ch (S. El-Khoury $\left.{ }^{a}\right)$, elvira.pirondinieepfl.ch (E. Pirondinib), martina.cosciadepfl.ch (M. Cosciab), silvestro.miceradepfl. ch (S. Micerab,c ${ }^{b}$, aude.billardeepfl.ch (A. Billard $\left.{ }^{\mathrm{a}}\right)$
}

Preprint submitted to Robotics and Autonomous Systems
sEMG-based systems were proposed for the estimation of hand and wrist movements, and consequently used as noninvasive interfaces for controlling exoskeletons [5, 6], prosthetic devices [7, 8, 9], computeranimated hands in a virtual environment [10], or for teleoperating robotic arms [9,11]. The previous studies focused on the investigation of discrete classifications of wrist abduction/adduction [9, 11], flexion/extension [7, 10, 12, 13] as well as of a different combination of finger motions [9, 11, 14]. Such strategies are useful for accomplishing power grasps that require simultaneous closure of all fingers on the object. However, this is insufficient to generate differentiated control of all fingers in the variety of pinch grasps used in dexterous objects manipulation, as required by the grasping of a larger variety of objects.

The differentiated control of all fingers is complex to achieve due to the high dimensionality of the hand's degrees of freedom. Indeed, the human hand is characterized by 21 degrees of freedom (DOFs) controlled by 29 muscles [15]. It has been hypothesized that humans are capable to control this large number of DOFs and use their hands dexterously thanks to a multidimensional reduction of the controlled variables operated by the central nervous system. This multidimensional re-

January 21, 2016 
duction may be accomplished through the use of postural synergies [16], corresponding to a number of hand postures that humans combine when grasping everyday life objects. Several works propose to exploit a mapping between upper limb EMG signals and hand postures [17, [18, 19, 20], as strategy to control the large number of the hand's degrees of freedom. However, in these approaches only the grasping phase, i.e. when the fingers have already reached their final configuration, was examined and the subjects were asked to perform the corresponding grasp keeping the upper-arm stalled. Nonetheless, the muscular activity differs between a static and a dynamic position of the arm. Moreover, during reaching-to-grasp movements, the configuration of the fingers and of the wrist changes simultaneously with the arms motion and this might influence the classification performance. This formation of the fingers, before reaching their final configuration, is defined as hand's preshape. The preshape of the hand is in direct relation with the characteristics of the object, specifically to the shape and width of it. In healthy human subjects, the hand's preshape occurs before the hand reaches the object, at around $60 \%$ of the reach and grasp motion [21]. Therefore, in order to accomplish a smooth control of the grasping gesture, it seems is crucial to classify the hand posture during the reaching phase before the occurrence of the preshape. Indeed, an accurate estimation of the final grasp posture in the early stages of the reach-to-grasp motion would ensure a faster reactivity of the assistive and the wearable devices. As result, these devices would increase their effectiveness and usability, and consequently increase the natural transition between the reaching and grasping phase on the prostheses increasing their acceptance by patients. However, at the moment only a limited number of studies focused on the detection of different grasp movements during reaching and grasping motions [22, 23, 24], and no measurement were performed to assess when a good classification was achieved respect to the hand's preshape .

In this this work we propose a new sEMG-based learning approach that decodes the grasping intention of the user at an early stage of the reach-to-grasp motion, i.e. before the final grasp/hand pre-shape takes place. We demonstrate as well the extensibility of our work to online applications. The rest of this paper is organized as follows. Section 2 describes the experimental set-up, protocol and methods used to record and analyze the data, while Section 3 reports the results of the experiments. In Section 4, the online robotic implementation is presented. In Section 5, we discuss the results of the experiment and the future perspectives.

\section{Methods}

\subsection{Participants}

Fourteen healthy young subjects (10 males and 4 females, average age $28.2 \pm 3.9$ ) participated in the experiment. All subjects were right handed according to the Edinburgh inventory test [25], and they had no prior history of neurological disorders and neuromuscular injuries. They performed the experiments with their dominant arm. The experiment was approved by the BMI Ethics Committee for Human Behavioral Research of the EPFL, and the recordings were carried out in agreement with the Declaration of Helsinki. All subjects gave written consent to the participation at the beginning of the experiment.

\subsection{Experimental Protocol}

The subjects were asked to reach and grasp 3 different objects, accounting for five different grasp types: precision disk, tripod, thumb-2 fingers, thumb-4 fingers, and ulnar pinch, see Figure (1). These grasps were mostly chosen for their common usage in daily life [26].

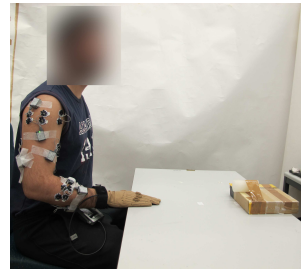

(a)

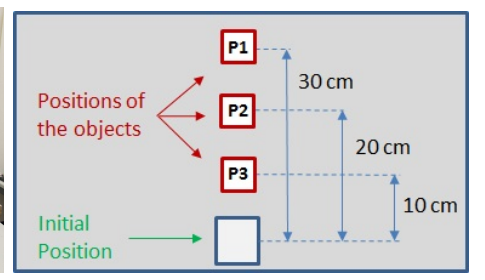

(b)
Figure 1: (a):The experimental setup showing the electrodes for EMG recording and the CyberGlove for capturing the hand joint angles, (b) the plan showing the initial position of the hand and the three positions of the object

During the experiment, the subjects seated in front of a table with the elbow flexed of about 90 and the hand placed on the table with the palm downward and the fingers pointing to the object, see Figure (1a). The subjects were asked to reach the object and grasp it with a predefined grasp type keeping the same hand's orientation for all the grasp types. The subjects were starting the self-paced motion after the advice of the experimenter and they had to declare that they grasped the object in order to consider the trial completed. The objects were placed at three different distances (i.e., $30 \mathrm{~cm}$, position $\mathrm{P} 1,20 \mathrm{~cm}$, position $\mathrm{P} 2$, and $10 \mathrm{~cm}$, position $\mathrm{P} 3$ ) from the initial hand's position, see Figure $(1 \mathrm{~b})$. All the fourteen subjects performed 20 trials for each of the five grasp types for position P1. After completing this first part of the experiment, six subjects continued the experiment 


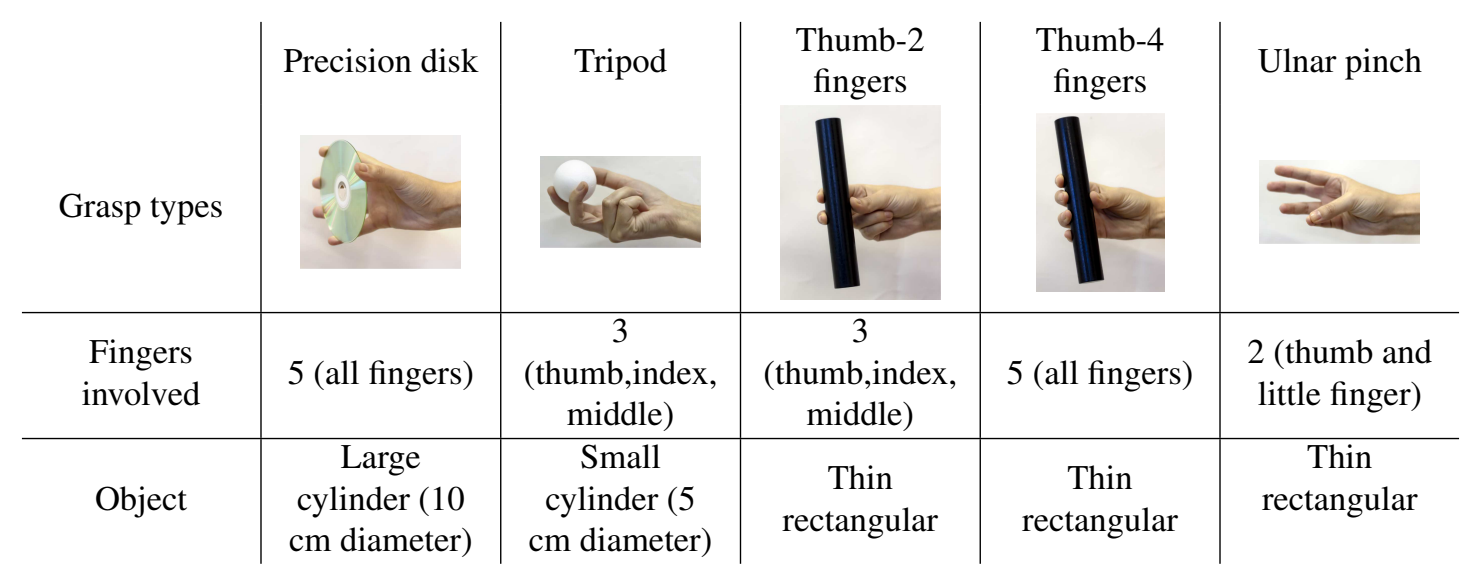

Table 1: Chosen grasp types [26].

for the positions $\mathrm{P} 2$ and $\mathrm{P} 3$ performing 15 trials for each grasp type. In addition, three subjects performed additionally fast reach-to-grasp motions for objects placed at position P1, asked to perform the motions by extending their arm with higher acceleration than the first part of the experiment. The subjects were performing all trials for one grasp before moving to the next grasp.

\subsection{Apparatus and pre-processing}

The EMG signals from 16 upper limb muscles (Table 2), were recorded using a Noraxon DTS desktop system, with a sampling rate of $1500 \mathrm{~Hz}$. The electrodes were placed, when it was possible, according to the standard procedure for surface electromyography for non-invasive assessment of muscles (SENIAM) guidelines [27]. At the beginning of the recordings, a manual test for the maximum voluntary contraction (MVC) was performed for each muscle. During the test, the subjects were asked to perform isometric contractions for each muscle. The test was repeated three times for each muscle, with a break after each contraction to prevent muscle fatigue.

The data were filtered with a seventh-order band-pass Butterworth filter between $50 \mathrm{~Hz}$ and $500 \mathrm{~Hz}$ for the suppression of movement artifacts. To construct a linear envelope, full-wave rectification was performed, followed by a smoothing with a low-pass seventh-order Butterworth filter with cutoff frequency at $20 \mathrm{~Hz}$. Finally, the resulting EMG signals were normalized by the MVC.

The joint angles of the fingers were measured using $\mathrm{Cy}-$ berGlove System's CyberGlove 1 with a sampling rate

\footnotetext{
${ }^{1}$ www.cyberglovesystems.com
}

\begin{tabular}{c|c} 
& Muscles \\
\hline 1 & Infraspinatus (INFRA) \\
2 & Deltoid Anterior (DANT) \\
3 & Deltoid Medial \\
4 & Deltoid Posterior (DPOS) \\
5 & Biceps Brachii long head (BICL) \\
6 & Triceps Brachii long head (TRIC) \\
7 & Brachialis (BR) \\
8 & Flexor Digitorum Superfialis (FLDS) \\
9 & Extensor Digitorum Communis (EXDC) \\
10 & Flexor Carpi Ulnaris (FLCU) \\
11 & Extensor Carpi Ulnaris (EXCU) \\
12 & Flexor Carpi Radialis (FLCR) \\
13 & Flexor Pollici Brevis (FLPB) \\
14 & Extensor Pollicis Brevis (EXPB) \\
15 & Adductor Pollicis Transversus (ADPT) \\
16 & Abductor Digiti Minimi (ABDM)
\end{tabular}

Table 2: Muscles which activity is captured for the reaching and grasping experiment.

of $280 \mathrm{~Hz}$. The Cyberglove has 22 bend sensors strategically located over the hand joints. Since bending can be detected anywhere along the sensor length, the glove can adapt well to different hands sizes and it needs to be calibrated in order to transform raw sensor values to hand joint angles. Linear regression was used to calibrate the 4 fingers (index, middle, ring, and little) while a data-driven approach was employed to model the nonlinear relationship between the thumb sensors and the joint angles. The recorded joint angles were used to compute the fingertip's position with respect to the wrist by forward kinematics. More details about the Cyberglove calibration procedure could be found in [28].

The two data streams were synchronized using a trigger 
signal provided by an Arduino board.

\subsection{Preshape Criteria}

As stated previously, the preshape of the hand is considered as the formation of the fingers before they reach their final configuration [29]. In this work, we employed two criteria to identify the occurrence of the hand preshaping [21],[30]. The first criterion was based on the distance between the fingertips of the thumb and the index finger, which are considered the fingers participating in most of the grasp types. For this reason they provide valuable information for the configuration of the fingers and more particularly for the opening and closing of the hand. In the case of the ulnar pinch, which is performed with the thumb and little finger, we replaced the index finger with the little finger. The first criterion is defined in the literature as the hands aperture and in this paper we will refer to it as aperture.

The second criterion was based on the estimation of the area of the polygon that is described by the fingertips involved in the grasp. For the precision disk and the thumb-4 fingers, the area considered was the one of the pentagon created by the fingertips of all five fingers. For the tripod grasp and the thumb-2 fingers, the area was the surface of a triangle defined by the fingertips of the thumb, index, and middle finger. Finally, for the ulnar pinch, the area was again the surface of a triangle but consisted of the fingertips of the thumb, the index finger, and the little finger. In the following section we will refer to this criterion as area.

To estimate the aperture and the area, we computed the position of the fingertips with respect to the wrist,using the joint angles of the fingers, recorded from the $\mathrm{Cy}$ berglove. The aperture and the area vary with respect to the opening and closing of the hand, providing objective information for the determination of the preshaping. In particular, the preshaping was assumed to correspond to the peak value of the aperture and the area while the grasp is considered complete when these criteria reach stability.

\subsection{Classification Method}

The preprocessed EMG signals were analyzed using a sliding time window of $150 \mathrm{msec}$ with an overlap of 50 msec. In order to embed the specificity of the motions time evolution, we combined an Echo State Network (ESN) [31] to classify the data with the Majority Vote (MV) criterion applied to each time window from the motion onset, as suggested in [32]. The MV criterion assigns a class label to the class that gathers the most votes. The preprocessed EMG data input were provided as input to the ESN, without extracting any feature from the EMG signals. Each ESN entailed 180 sigmoid units, with a transfer function $f=t a n h$, and it outputted 5 classes for each of the 5 grasp types. Moreover, the activation function of the output units was chosen to be the identity function. The classification for each time window was fed to the MV algorithm, where each vote corresponded to the result of the classification.

Since the classification strategy is implemented online, the following analysis was performed in the time domain, avoiding time normalization. As the hand's preshape occurs around the $60 \%$ of the reaching motion, we chose to analyze the first second of the reaching motion. This approach enables the capture of the hand's preshape in different time steps and its the relation with the classification rates.

One classification machine was trained from every subject. For the first position of the object (P1), the classification machine was built with the $75 \%$ of the dataset of 100 trials (i.e., training dataset) and tested in the remaining data (i.e., 25\%) using the cross-validation method. For the generalization over different distances (i.e., positions P2 and P3), two different classification machines were built. In the first case, the training dataset was constituted by the data from two different positions, and the testing dataset included only the data from the remaining positions. In the second case, instead, the machine was trained on one position and tested in the other two. Moreover, in the case of the trials at different speeds, we tried different combination of training and testing data in order to examine the performance of generalization. First, the classification machine was trained with data of self-paced motions and tested with data on fast motions. As a second step, the classification machine was trained with data of fast motions and tested with data of selfpaced motions. In the last test, we mixed the data of fast and slow motions while the classification machine was trained with $75 \%$ of the data and tested with the remaining $25 \%$, following a four-folder cross-validation.

\subsection{Online Robotic Implementation}

For the purposes of the online implementation, we used a right Allegro hand from Simlab ${ }^{2}$ This is a humanoid hand with 16 DOF split equally on 4 fingers. While it is only an approximate reproduction of the dexterity of the human hand and it has more DOFs than currently available assistive devices, it serves as a benchmark for our ability to reproduce grasps with similar dexterity to those generated by humans. The Allegro

\footnotetext{
${ }^{2}$ http://www.simlab.co.kr/Allegro-Hand.htm
} 


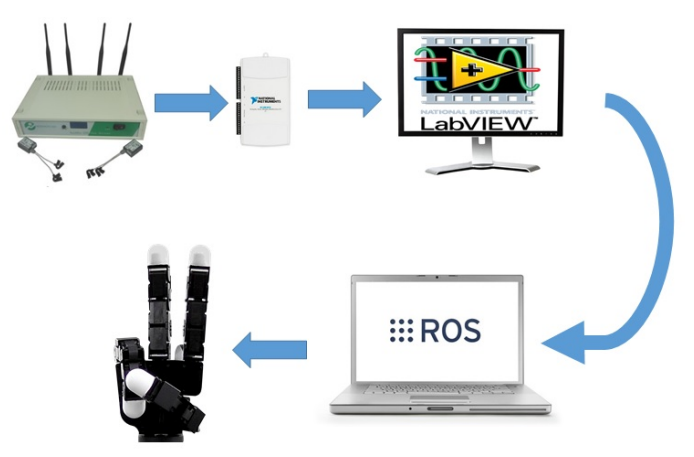

Figure 2: Control scheme of the robotic implementation.

hand has also the advantage to be controlled at an extremely fast rate $(400 \mathrm{~Hz})$ that enables demonstrating the benefit of our early pre-shape detection for real-time control of finger closure during the arm movements.

In the online robotic implementation, the EMG signals were acquired using a National Instruments USB-6210 data acquisition board with a sampling rate at $1000 \mathrm{~Hz}$. The acquired signals were pre-processed (filtered and rectified as described in previous subsection) using $\mathrm{Na}$ tional Instruments LabView 2014 installed in a desktop computer (Intel Xeon @ $2.27 \mathrm{GHz}$ with Windows 8.1). The pre-processed data were streamed to a portable computer (Inteli7 @ 2.6 GHz with Ubuntu 14.01) and classified. Finally, the corresponded joint angles were imported to the Allegro hand using ROS. The straight forward control scheme is presented in the Figure (2)).

\section{Results}

In order to assess the classication accuracy and the robustness of the results, we defined the success rate as the percentage of movements correctly classified for a specific grasp type on the total number of reach-to-grasp motions corresponding to that specific grasp type (Recall column in Table (3)). We also computed the precision measure (i.e., the percentage of trials correctly classified for a specific grasp type on the total number of reach-to-grasp movements classified to the same grasp type) and the F-measure, which corresponds to the harmonic average of the recall and precision values, for each grasp type. A F-measure score of 1 means that each motion belonging to a specific grasp type was perfectly classified as such. After presenting the Recall, Precision and F-measure values of each time window, we define the classification performance as the number of correctly classified trials over the total number of trials, for simplicity purposes.

\subsection{Reach-to-Grasp classification strategy}

Table (3) shows the average and standard deviation of the classification results across subjects and grasps for 10 different time windows starting from 0.15 to 1.05 seconds with a step size of $100 \mathrm{msec}$. The average classification performance among subjects increased during time, as the hand was getting closer to the object (see Figure (3p) for all the three positions with a slight decrease only at 450 and $950 \mathrm{msec}$ for P1 (see Table (3)). In particular, a success rate of $90 \pm 4.5 \%$ was reached $0.5 \mathrm{sec}$ after motion's onset (i.e., half-way through the reaching motion). On average, a F-measure of 0.91 was obtained for the five grasp types and for all time windows. However, it was higher than 0.76 already $150 \mathrm{msec}$ after motion onset, showing that an accurate classification of the five grasp types was possible before the grasp occurred.

\begin{tabular}{|c|c|c|c|}
\hline & \multicolumn{3}{|c|}{ ESN MV } \\
\hline time $(s)$ & Precision & Recall & F-measure \\
\hline 0.15 & $0.81 \pm 0.06$ & $0.84 \pm 0.05$ & $0.76 \pm 0.08$ \\
\hline 0.25 & $0.89 \pm 0.04$ & $0.91 \pm 0.04$ & $0.88 \pm 0.05$ \\
\hline 0.35 & $0.87 \pm 0.06$ & $0.89 \pm 0.06$ & $0.84 \pm 0.06$ \\
\hline 0.45 & $0.93 \pm 0.04$ & $0.94 \pm 0.04$ & $0.92 \pm 0.04$ \\
\hline 0.55 & $0.91 \pm 0.06$ & $0.92 \pm 0.05$ & $0.90 \pm 0.06$ \\
\hline 0.65 & $0.95 \pm 0.02$ & $0.96 \pm 0.02$ & $0.94 \pm 0.03$ \\
\hline 0.75 & $0.95 \pm 0.03$ & $0.95 \pm 0.03$ & $0.94 \pm 0.03$ \\
\hline 0.85 & $0.97 \pm 0.02$ & $0.97 \pm 0.01$ & $0.96 \pm 0.02$ \\
\hline 0.95 & $0.96 \pm 0.03$ & $0.97 \pm 0.02$ & $0.95 \pm 0.03$ \\
\hline 1.05 & $0.97 \pm 0.02$ & $0.98 \pm 0.01$ & $0.96 \pm 0.02$ \\
\hline Total av. & 0.92 & 0.93 & 0.91 \\
\hline
\end{tabular}

Table 3: The average and standard deviation of the classification results across grasp types and subjects. The Recall values correspond to the percentage of EMG data correctly classified as a specific grasp type to the total number of reach-to-grasp motions corresponding to the same grasp type. The Precision values correspond to the percentage of EMG data correctly classified as a specific grasp type to the total number of reach-to-grasp motions classified to the same grasp type. The $F$-measure values corresponding to the harmonic average of the recall and precision values. The last row of each of the above tables correspond to the total average across time windows.

Figure (3a) shows the confusion matrix averaged across subjects for the five grasp types $550 \mathrm{~ms}$ after motion onset. This timing was chosen because it corresponded to half-way of the reach-to-grasp motion. Precision disk, tripod and ulnar pinch were distinguishable already at half motion $(89.5 \%, 92.7 \%$ and $98.3 \%$ respectively), while thumb-2 fingers and thumb-4 fingers were distinct later in the reaching motion when the hand was closer to the object( $85.1 \%$ for both of the classes). As expected from the hand configuration during the grasping, a misclassification tended to occur between tripod and precision disk and between thumb-2 and thumb- 


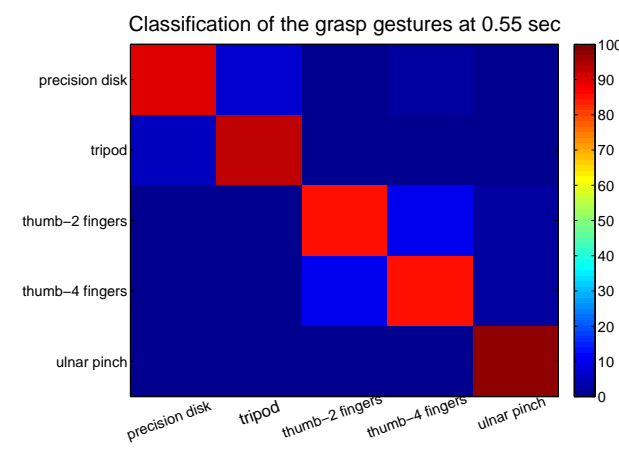

(a)

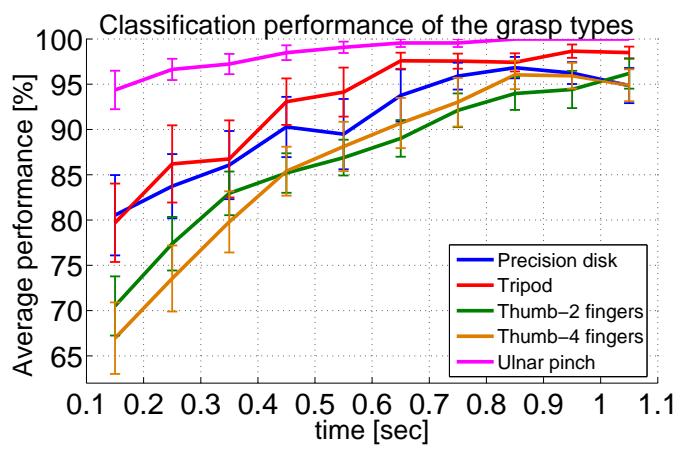

(b)

Figure 3: a) Confusion matrix of the classification between grasp types in among the subjects, $0.55 \mathrm{sec}$ after the onset of the motion. Warmer color indicates higher classification performance, b)Average and standard error of the classification performance of all the grasp types among subjects for $30 \mathrm{~cm}$ distance of the object.

4 fingers $(85.5 \%$ and $85.8 \%$ respectively). From Figure (3p), we notice that thumb-2 fingers and thumb-4 fingers are reaching $90 \%$ of classification rate $0.7 \mathrm{sec}$ after the onset of the motion.

\subsection{Classification rate Vs Hand preshape}

We estimated the occurrence of the hand's preshape with 1) the aperture: the distance between the fingers involved during the grasp (i.e., thumb and index finger for Precision disk, Tripod, Thumb-2 fingers and Thumb-4 fingers and thumb and pinky finger for Ulnar pinch grasp), and 2) the area: the surface obtained interconnecting the tips of the fingers involved in each grasp. Figure (4) shows the average values of these two criteria across all subjects for the five grasp types separately. The first criterion, hand's aperture, is depicted with blue color while the second criterion, the corresponded area, is presented with the red curve. The green vertical line indicates the average time across subjects and repetitions where a classification success rate above $90 \%$ was obtained. It is worth mentioning that these criteria define the occurance of the hand's preshape by their peak, i.e the moment they reach their maximum value.

Except for thumb-2 and thumb-4 fingers, a classification performance of $90 \%$ before the pre-shape of the hand. Indeed, the average classification time across trials and subjects for the precision disk grasp was $0.55 \mathrm{sec}$, while the pre-shape of the hand occurred at the peaks of the two criteria between 0.6 and $0.9 \mathrm{sec}$. For the ulnar pinch, a classification ratioe of $90 \%$ was obtained at $0.15 \mathrm{sec}$, while the pre-shape of the hand occurred at $0.3 \mathrm{sec}$. Similarly, for the tripod grasp, a good classification was obtained before the pre-shape of the hand at $0.8 \mathrm{sec}$. Finally, for the thumb-2 fingers and the thumb-4 fingers, the computed hand pre-shape criteria did not indicate a clear preshaping for all subjects. Indeed, the

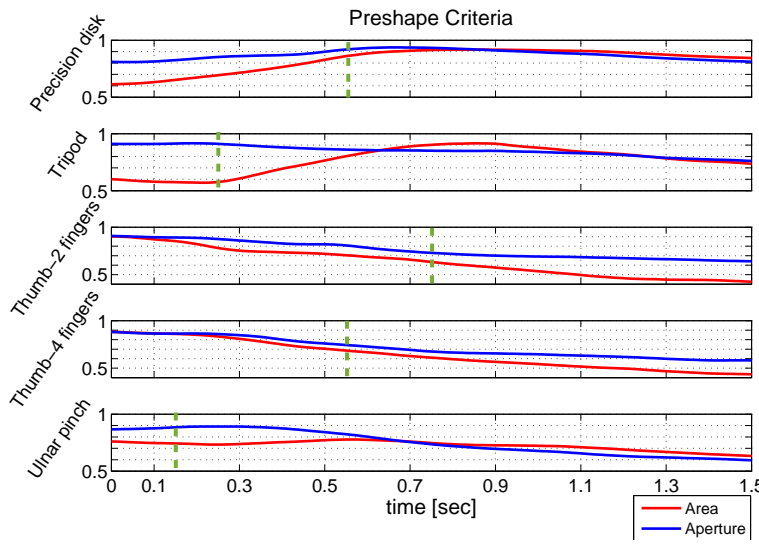

Figure 4: The average value of preshpe criteria across all subjects: the blue curve corresponds the hand aperture criterion while the red curve corresponds to the area criterion. The vertical green line indicates a classification rate of $90 \%$.

two criteria did not always show a peak during the motion. Although a classification success of $90 \%$ occured at $0.75 \mathrm{sec}$ and $0.55 \mathrm{sec}$ for these two grasp respectively, it is difficult to perform a relation with the hand's preshape. This could happen due to hand's initial position and the object's width. Indeed, the initial position of the hand was flat with the fingers extended while the task was grasping a thin object. This leaded to a constant flexion of the fingers through the reaching motion without fingers' extension as visible also from the continuous decrease of the values of the two criteria.

\subsection{Generalization on different distances}

In order to test the generalizability of our classification approach, we tested the results of the classification across different positions of the objects. Initially, we applied the classification taking into account, in the 


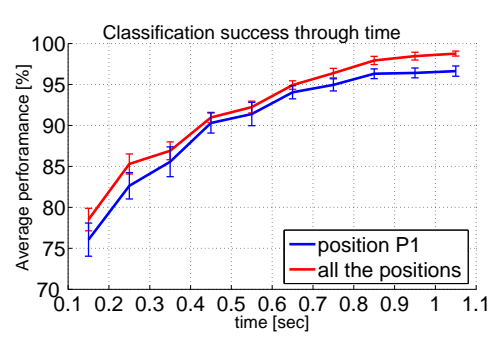

(a)

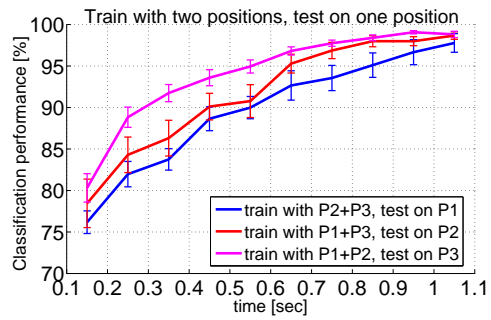

(b)

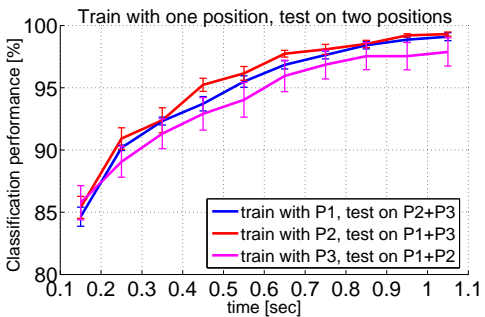

(c)

Figure 5: Classification success rate in different distances: (a) Average classification performance and standard error on the reaching motion: The blue line presents the performance of the classification of grasp types on position P1. The red line corresponds to the classification performance when all the positions (P1, P2 and P3) are taken into account, (b) Average classification performance and standard error on training with two positions and testing on the third: The blue line presents the performance when training on reaching motions to two positions P2 and P3 and testing on position P1. Respectively, the red line corresponds to training on positions $\mathrm{P} 1$ and $\mathrm{P} 3$ and testing on position P2 while the magenta line corresponds to training on positions P1 and P2 and training on position P3, (c) Average classification performance and standard error on training with one position and testing on the other two positions: The blue line presents the performance when training with reaching motions to positions $\mathrm{P} 1$ and testing on positions $\mathrm{P} 2$ and $\mathrm{P} 3$. Respectively, the red line corresponds to training on position P2 and testing on positions $\mathrm{P} 2$ and $\mathrm{P} 3$, while the magenta line corresponds to training on positions P3 and training on position P1 and P2.

training, the reaching motions for all objects' positions (i.e., P1, P2 and P3). The classification performance was similar to the one obtained training and testing the classifier only considering position P1 (see Figure (5a)). The $90 \%$ of accuracy was achieved $0.5 \mathrm{sec}$ from the motion onset for all the three positions, when the classifier was trained using all motion distances.

As second step, we examined the generalization across distances by i) training the classifier on two positions (i.e., P1 and P2 or P1 and P3) and testing it on the remaining third position (P3 or $\mathrm{P} 2$, respectively) and, ii) training the classifier on one position (i.e., $\mathrm{P} 1$ or $\mathrm{P} 2$ or P3) and testing it on the other two (i.e., P2 and P3 or P1 and $\mathrm{P} 3$ or $\mathrm{P} 1$ and $\mathrm{P} 2$, respectively). The performances were higher when the classifier was trained with a single position with respect to a classifier trained on two positions (see Figures (5b) and (5k)). Indeed, the average performance at $0.45 \mathrm{sec}$ after movement onset was $93.93 \pm 1.7 \%$ and $90.78 \pm 2.5 \%$ when training on one position and on two positions, respectively. For the classifier trained with two positions, the performance was better when the training set included movements from farthest distances (i.e., P1 and P2) and the testing set included the shortest distance (i.e., P3) (see Figure (5b)). The performance in this case was $93.6 \pm 3.6 \%$ after $0.45 \mathrm{sec}$ from the motion on set. For the classifier trained with a single position, instead, the best classification performance was achieved when the classifier was trained in the middle distance (i.e., P2) and tested on the other two distances (i.e., P1 and P3) (see Figure (5k)). In this case, a classification accuracy of $95.2 \pm 2.0 \%$ was achieved $0.45 \mathrm{sec}$ after movement onset. It is worth mentioning that this case (i.e., training with P2 and testing on P1 and P3) presented as well the smallest standard error when compared to the other gen- eralizing

\subsection{Speed effect}

In order to further evaluate the generalizability of our approach, we examined the effect of the speed on the classification. We first analysed the differences in fingers' motion in fast movements with respect to self pace motions. As expected there was a significant difference on the timings of hand opening and closing between motions performed at self-paced speed and at fast speed (see Figure (6a)). Indeed, the subjects opened and closed their hand in fast motions sooner in time than in self-paced motions.

As first step, we mixed the data at self pace and during fast motions for training and testing. The classification performance reached $90 \pm 2.3 \%$ of success after $0.55 \mathrm{sec}$ from movement onset. We then compared this first classification with the results obtained training the classifier in the fast motions and testing it in either for the fast or self pace motions, or training the classifier with the movements at self-selected speed and testing it for the fast motions. As expected when using only data from the fast motions both for training and testing, the classification performance reached an accuracy higher than $90 \%$ earlier than when mixing the movements $(0.35 \mathrm{sec}$ for fast motions, $0.55 \mathrm{sec}$ for self-paced motions). On the contrary, the classifiers trained and tested in different datasets achieved lower performances than that trained and tested with mixed data. In particular, the accuracy of $90 \%$ was achieved $0.7 \mathrm{sec}$ after motion onset. Moreover, the standard error of these classifiers was higher than $4.5 \%$, which indicated that the classifier performed significantly better in some subjects than in others. 


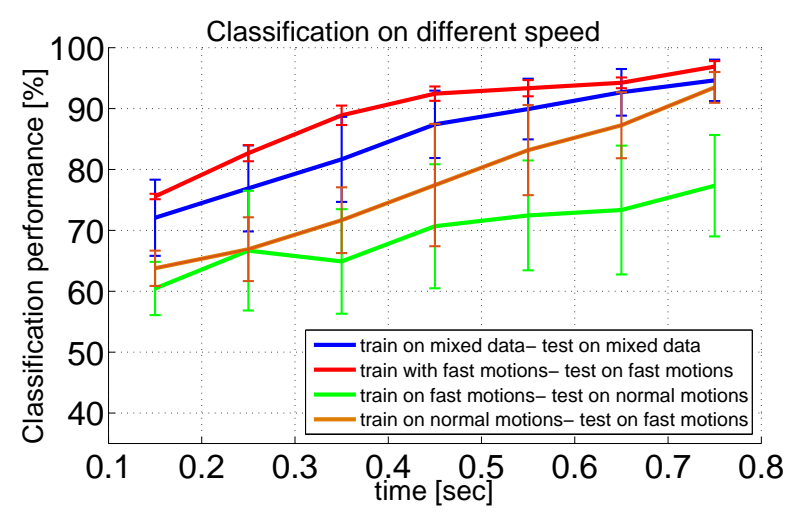

(a)

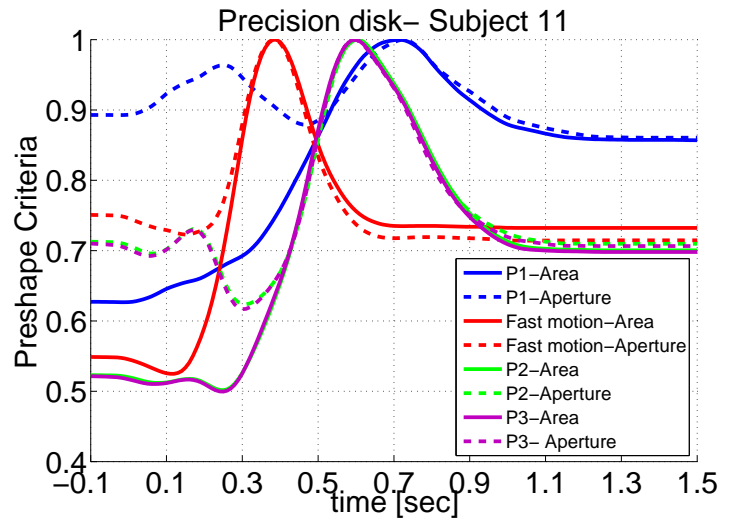

(b)

Figure 6: a: Average classification performance and standard error on the fast motions: The red line presents the performance of the classification of grasp types with the data of fast motions. The blue line corresponds to the classification performance when the data of fast motions were mixed with the data of normal motions. The green line corresponds to the performance when training with fast motions and testing on normal motions. The brown line corresponds to the performance when training with normal motions and testing on fast motions. the magenta and cyan vertical lines indicate the peak of the preshape criteria then subject 11 performed the precision disk grasp with fast and normal motions respectively as depicted in Figure 63). b: Preshape criteria on the precision disk of subject 11: The subject opens its fingers sooner in fast motions than it does in normal motions.

\subsection{Online Robotic Implementation}

In order to demonstrate the usability of the proposed approach for the estimation of the final grasp gesture in the early stages of the reaching motion for an assistive or a rehabilitative application, we present here an online robotic implementation of our approach. The system was trained offline while the testing was performed online using the aforementioned control scheme of the Figure (2)). The corresponded joint angles of the predicted class were imported to the Allegro hand after $0.5 \mathrm{sec}$, as the classification performance reached above $90 \%$ after $500 \mathrm{msec}$ as noticed in the aforementioned analysis. Moreover, in order to avoid a continuous change of the robotic fingers' configuration, we added a threshold of $1 / 3$ on the confidence on the outcome of the majority vote. The confidence of the majority vote was defined as the difference in votes of the winner from the second winner divided by the sum of all the votes.

Three healthy subjects participated in the online implementation experiment(two males and one female). The subjects performed 15 reach-to-grasp motions for each grasp type with the objects placed $30 \mathrm{~cm}$ away from the initial position of the hand. The recorded dataset consisted of 75 trials was used to train the system offline. After the training phase, the subjects performed 30 reach-to-grasp motions for different objects. The results of the online implementation are presented on the Table 4 The $70.8 \%$ of the trials the were successful allowing the execution of the correct grasp type (see the video to lasa.epfl.ch/ sina/Demo.mp4 and

\begin{tabular}{|c|c|c|c|}
\hline Subject & Total trials & Successful trials & Preceded \\
\hline 1 & 30 & 20 & 14 \\
\hline 2 & 30 & 22 & 15 \\
\hline 3 & 30 & 22 & 14 \\
\hline
\end{tabular}

Table 4: The results of the online implementation. The third column shows the number of successful trials, meaning that the robotic hand performed the same grasp type with the subject. The forth column shows the number of trials that the robotic hand reached its final configuration before the subject reached the object.

Figure (7p). In the $67.2 \%$ of the successful trials the robotic hand reached its final configuration before the subject reached the object.

\section{Discussion}

Previous studies [23, 17, 34], presented different approaches for mapping EMG signals to reaching and grasping motions according to object's features and locations and during static or dynamic gestures. However, no one correlated the classification performance of the reach-to-grasp motion to the time of fingers/hand preshape. An early decode of the grasping type is important as it would enable the wearable device to react promptly to the intention of the user. Therefore, we here proposed an EMG-based learning approach that decodes the grasping intention of the user at an early stage of the reach to grasp motion. Our approach was based on an Echo State Network (ESN) [31] combined with a Majority Vote (MV) criterion applied to time windows of $150 \mathrm{~ms}$ from the motion onset to the grasp of the object. 


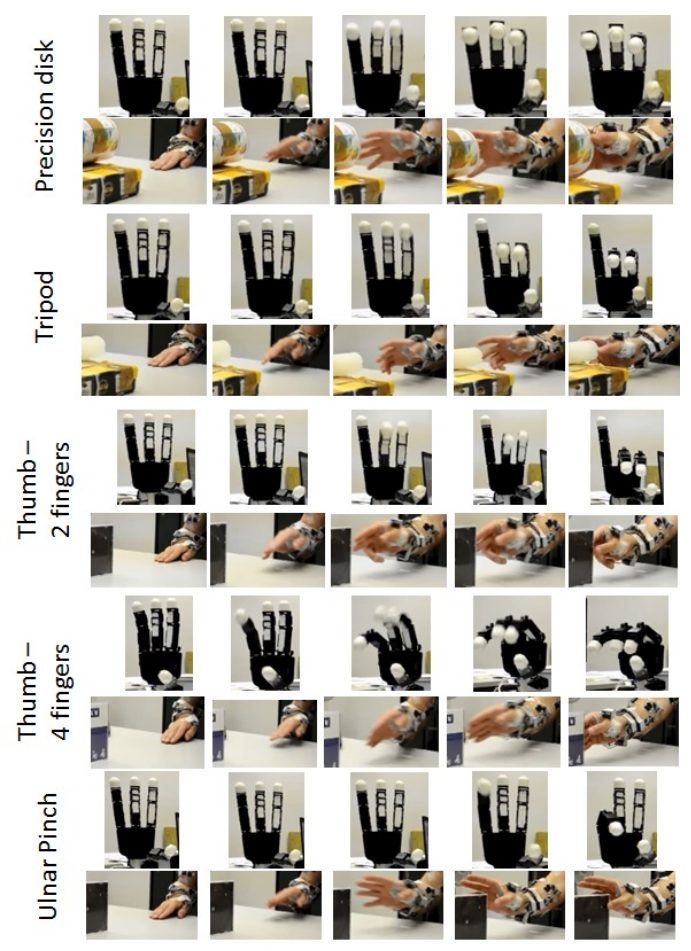

Figure 7: Snapshots of the finger motions of the robotic hand and human hand.

We applied the algorithm to an offline classification of five different grasp types: precision disk, tripod, ulnar pinch, thumb-2 fingers and thumb-4 fingers grasp. Furthermore, we examined whether the proposed approach was robust to different objects' location and to different motion speeds. Indeed, the object's distance and the movement velocity could differentiate the activation of the muscles, especially for the muscles of the upper arm, influencing the classification performance. Finally, we demonstrated in three subjects the usability of our approach for the online control of the Allegro hand.

It was shown that the hand's pre-shaping occurs from $60 \%$ to $80 \%$ of the reach-to-grasp motion, which corresponds to the time instant when the distance between the thumb and the index reached its maximum [21], [30]. Our results confirmed that hand's preshape occurs after the $50 \%$ and is in direct relation with the object's size and fingers' initial configuration. Furthermore, the detection of the preshape becomes more obvious when the task demands extension of the fingers. On the other hand, when reaching to grasp a thin object, the detection of the hand's preshape becomes more difficult and more criteria may be necessary for the detection of the hand's preshape.

Moreover, it has been suggested that a period between the $25 \%$ and the $50 \%$ could be sufficient to obtain dif- ferences in muscle activity when reaching to grasp three different objects [35]. Expanding this suggestion, our results showed that it is possible to classify five grasp types using EMG data from early stages of the reaching motion. Relating the classification performance with the hand's preshape, our offline results showed that a classification rate of $90 \%$ was achieved before the hand preshaping for the precision disk, tripod, and ulnar pinch. For the thumb-2 fingers and the thumb-4 fingers grasps, instead, the computed hand preshape criteria did not always show a peak, thus the hand preshaping was not always clearly detected.

In addition, we examined the proposed approach when the object is placed in different distances. Our results showed no significant differences between the classification performance on different object's distances. This outcome may be explained from the training of the classifier in time steps without normalizing the time. With this approach, the classifier gained a level of tolerance in different muscle activation. Furthermore, we evaluated different combinations of training and testing dataset acquired at different object's distances and we concluded that the best performance was achieved when the classifier was trained in the middle distance and tested in distances $10 \mathrm{~cm}$ larger or shorter. A potential expansion of our tests would be the inclusion of different positions in space.

Although no significant differences were found when considering different object's distances, as the motion velocities in self-paced motions may be slightly different depending on the object's location, fast motion influenced significantly the classification performance. In particular, the rapid activation of the arm muscles during fast motions differentiates the EMG signals that resulted in a lower performance when significantly different velocities were taken into account. A classifier that included different speed motions performed sufficiently well only when the training involved both data form normal and fast motions. The reduction of the performance obtained when the classifier was trained with a single speed motion may cause inconvenient behavior for assistive and prosthetic devices. Therefore, higher attention should be paid to the effect of speed on the design of more human-friendly and convenient robotic devices that should interact with patients. Indeed, comfort and robot-human interfaces represent two pivotal aspects in robotic-rehabilitation approaches.

For a proof of concept, we integrated our approach in the control of an Allegro hand. After a training phase performed offline including 15 repetitions for each object of the five grasp types, the success rate of classification was around $70.8 \%$. The difference between the 
offline and online classification performance could be due to different sampling frequency used in the online implementation. Although the decrease of the performance, this robotic implementation leads to the conclusion that the early estimation of the final grasp from the EMG signals could be applied to a robotic system and, as an extension, may be applied to the control of a prosthetic device or of an exoskeleton. Additional works could be done to integrate to the robotic system tactile sensors for the attainment of a safe/stable grasp. Indeed, recent developments on the sensory field [36, 37], showed that the design of compliant prosthesis should also include the sensory feedback to the user. This feedback could involve visual and tactile information in order to provide a compliant solution to the demands of the different conditions. A control scheme of an upper arm prosthesis that combines a variety of sensors (e.g EMG, vision, tactile sensors) would provide a robust use of the device. Moreover, a potential next step would be to include the proposed approach into the control of a fingers' exoskeleton in order to examine any inconvenience that this may cause and be further developed on a semi-autonomous control scheme.

Moreover, it has been reported [24] that it possible to relate the object's characteristics with the muscular activity. Towards this direction, the decode of the preshape by the EMG signals, as presented in our work, could provide valuable information for object before the contact of the fingers with the object. As this information comes in advance of the contact, it could be potentially used for accomplishment of a safe grasp.

Finally, a further interesting extension of this work is the classification of specific finger configuration with different hand orientations. Indeed, during grasping different orientations of the hand are used. Studies [38, 39] has reported a successful classification of the simultaneous motion of the wrist and fingers. A potential combination of the decode of the grasping intention of the user with the simultaneous control of the wrist will provide a more natural motion of the wearable device with respect to the human motion.

\section{Conclusion}

In this paper we propose an electromyographic based approach for decoding the grasping intention in reachto-grasp motions. Our results have shown that it is possible to decode the grasping intention in the early stages of the reaching motion during the hand's preshape, i.e. before the fingers final configuration. This is very important for proper reaction of the wearable device and the increase of the natural motion of a prosthesis. Further extensions should involve the evaluation of the proposed approach in different hand's orientation and different object's positions in space.

\section{Acknowledgment}

This work was supported by the Swiss National Science Foundation through the National Centre of Competence in Research in Robotics and by the Bertarelli Foundation.

\section{References}

[1] O. Lambercy, L. Dovat, H. Yun, S. K. Wee, C. WK Kuah, K. SG Chua, R. Gassert, T. E. Milner, C. L. Teo, E. Burdet, Effects of a robot-assisted training of grasp and pronation/supination in chronic stroke: a pilot study, in Journal of NeuroEngineering and Rehabilitation, vol. 8, no. 63, 2011.

[2] E. Biddiss, Need-Directed Design of Prostheses and Enabling Resources, in Amputation, Prosthesis Use, and Phantom Limb Pain: An Interdisciplinary Perspective, 2010.

[3] P. Maciejasz, J. Eschweiler, K. Gerlach-Hahn, A. Jansen-Troy, S. Leonhardt, A survey on robotic devices for upper limb rehabilitation, in Journal of NeuroEngineering and Rehabilitation, vol. 11, no. 3, 2014.

[4] D. Novak, R. Riener, A survey of sensor fusion methods in wearable robotics, in Robotics and Autonomous Systems, 2014.

[5] Z. O. Khokhar, Z. G. Xiao, and C. Menon, Surface EMG pattern recognition for real-time control of a wrist exoskeleton, in BioMedical Engineering OnLine, vol. 9, pp. 41-57, 2010.

[6] A. Ziai and C. Menon, Comparison of regression models for estimation of isometric wrist joint torques using surface electromyography, in Journal of NeuroEngineering and Rehabilitation, vol. 8, pp. 56-67, 2011.

[7] D. Nishikawa, W. Yu, H. Yokoi, and Y. Kakazu, EMG Prosthetic Hand Controller using Real-time Learning Method, in Proceedings of the IEEE International Conference on Systems, Man, and Cybernetics, (SMC), 1999.

[8] Z. Ju, H. Liu, Human Hand Motion Analysis With Multisensory Information, in IEEE/ASME Transactions on Mechatronics, vol. 19, no. 2, 2014.

[9] O. Fukuda, T. Tsuji, M. Kaneko, and A. Otsuka, A HumanAssisting Manipulator Teleoperated by EMG Signals and Arm Motions, in IEEE Transactions on Robotics and Automation, vol. 19, no. 2, 2003.

[10] F. Sebelius, M. Axelsson, N. Danielsen, J. Schouenborg and T. Laurell, Real-time control of a virtual hand, in Technology and Disability, vol. 17, pp. 131-141, 2005.

[11] P. Shenoy, K. J. Miller, B. Crawford, and R. P. N. Rao, Online Electromyographic Control of a Robotic Prosthesis, in IEEE Transactions on Biomedical Engineering, vol. 55, no. 3, 2008.

[12] Y. Huang, K. B. Englehart, B. Hudgins, and A. D. C. Chan, A Gaussian Mixture Model Based Classification Scheme for Myoelectric Control of Powered Upper Limb Prostheses, in IEEE Transactions on Biomedical Engineering, vol. 52, no. 11, 2005.

[13] K. Kita, R. Kato, H. Yokoi, and Tamio Arai, Development ofAutonomous Assistive Devices-Analysis of change of human motion patterns, in IEEE International Symposium on Robot and Human Interactive Communication (RO-MAN), 2006. 
[14] M.W.Jiang, R.C. Wang, J.Z. Wang, and D.W.Jin, A Method of Recognizing Finger Motion Using Wavelet Transform of Surface EMG Signal, in Proceedings of the IEEE Engineering in Medicine and Biology 27th Annual Conference, 2005.

[15] L. A. Jones and S. J. Lederman, Human Hand Function, in Oxford University Press, 2006.

[16] M. Santello, M. Flanders, and J. F. Soechting, Postural hand synergies for tool use, The Journal of Neuroscience, 1998.

[17] S.A. Dalley, H.A. Varol, M. Goldfarb, A Method for the Control of Multigrasp Myoelectric Prosthetic Hands, in IEEE Transactions on Neural Systems and Rehabilitation Engineering, vol. 20, no. 1, 2012.

[18] C. Sapsanis, G. Georgoulas, A. Tzes, and D. Lymberopoulos, Improving EMG based Classification of basic hand movements using EMD, in Proceedings of the IEEE Engineering in Medicine and Biology 35th Annual Conference, 2013.

[19] G. Ouyang, X. Zhu, Z. Ju, and H. LiuDynamical Characteristics of Surface EMG Signals of Hand Grasps via Recurrence Plot, in IEEE Journal of Biomedical and Health Informatics, vol. 18, no. $1,2014$.

[20] R. J. Smith, F. Tenore, D. Huberdeau, R. Etienne-Cummings, and N. V. Thakor, Continuous Decoding of Finger Position from Surface EMG Signals for the Control of Powered Prostheses, in Proceedings of the IEEE Engineering in Medicine and Biology 30th Annual Conference, 2008.

[21] Y. Paulignan, C. MacKenzie, R. Marteniuk, and M. Jeannerod, The coupling of arm and finger movements during prehension, Exp Brain Res, vol. 79, pp. 431-435, 1990.

[22] J. Gonzlez, Y. Horiuchi and W. Yu, Classification of Upper Limb Motions from Around-Shoulder Muscle Activities: Hand Biofeedback, in the Open Medical Informatics Journal, 2010.

[23] M. V. Liarokapis, P. K. Artemiadis, K. J. Kyriakopoulos, and E. S. Manolakos, A Learning Scheme for Reach to Grasp Movements: On EMG-Based Interfaces Using Task Specific Motion Decoding Models, in IEEE journal of Biomedical and Health Informatics, vol. 17, no. 5, 2013.

[24] N. Fligge, H. Urbanek, and P. van der Smagt, Relation between object properties and EMG during reaching to grasp, in Journal of Electromyography and Kinesiology, vol. 23, pp. 402410, 2013.

[25] R. C. Oldfield, The Assessment and Analysis of Handedness: the Edinburgh Inventory, in Neuropsychologia, vol. 9, pp. 97-113, 1971.

[26] I. M. Bullock, J. Z. Zheng, S. De La Rosa, C. Guertler, and A. M. Dollar, Grasp Frequency and Usage in Daily Household and Machine Shop Tasks, in IEEE Transactions on Haptics, vol. 6, no. 3, 2013.

[27] H. J. Hermens, et al., Development of recommendations for SEMG sensors and sensor placement procedures, J Electromyogr Kinesiol, vol. 10, no. 5: p. 361-74, 2000.

[28] R.L. de Souza, R. L., S. El-Khoury, J. Santos-Victor, and A. Billard, Towards comprehensive capture of human grasping and manipulation skills, in proceedings of the Thirteenth International Symposium on the 3-D Analysis of Human Movement, 2014.

[29] P. Haggard, A. Wing, Coordinated responses following mechanical perturbation of the arm during prehension, Exp Brain Res., vol 102, p. 483-494, 1995.

[30] T. Supuk, T. Kodek, T. Bajd, Estimation of hand preshaping during human grasping, Medical Engineering \& Physics, vol. 29, pp. 790-797, 2005.

[31] H. Jaeger, The "echo state" approach to analyzing and training recurrent neural networks, GMD Report 148, GMD - German National Research Institute for Computer Science, 2001.

[32] K. Englehart, B. Hudgins, A robust, real-time control scheme for multifunction myoelectric control,in IEEE Transactions on Biomedical Engineering, vol. 50, no. 7, 2003.

[33] F. P. Kendall, E. Kendall McCreary, P. Geise Provance, M. McIntyre Rodgers, W. A. Romani, Muscles Testing and Function with Posture and Pain, 5th edition, published by Lippincott Williams \& Wilkins, 2005.

[34] J, Carpaneto, K.H. Somerlik, T.B. Krueger, T. Stieglitz, S. Micera, Natural muscular recruitment during reaching tasks to control hand prostheses, IEEE RAS \& EMBS International Conference on Biomedical Robotics and Biomechatronics, 2012.

[35] S. Martelloni, J. Carpaneto and S. Micera, Characterization of EMG Patterns From Proximal Arm Muscles During Object- and Orientation-Specific Grasps, IEEE Transactions On Biomedical Engineering, vol. 56, p. 2529-2536, 2009.

[36] S. Raspopovic, M. Capogrosso, F. M. Petrini, M. Bonizzato, J. Rigosa, G. Di Pino, J. Carpaneto, M. Controzzi, T. Boretius, E. Fernandez, G. Granata, C. M. Oddo, L. Citi, A. L. Ciancio, C. Cipriani, M. C. Carrozza, W. Jensen, E. Guglielmelli, T. Stieglitz, P. M. Rossini, S. Micera Restoring Natural Sensory Feedback in Real-Time Bidirectional Hand Prostheses, Science Translational Medicine, vol. 6, no. 222, 2014.

[37] D. Novak, X. Omlin, R. Leins-Hess, and R. Riener, Effectiveness of different sensing modalities in predicting targets of reaching movements, International Conference of the IEEE EMBS, 2013

[38] A. J. Young, L. H. Smith, E. J. Rouse, L. J. Hangrove, Classification of Simultaneous Movements using Surface EMG Pattern Recognition, IEEE Transactions on Biomedical Engineering, vol. 60(5), 2013.

[39] A. Fougner, O.yvind Stavdahl, P. J. Kyberd, System Training and Assessment in Simultaneous Proportional Myoelectric Prosthesis Control, Journal of Neuroengineering and Rehabilitation, vol. 11:75, 2014. 